\title{
Special issue: Rising Stars in Polymer Science 2021
}

\author{
Keiji Tanaka ${ }^{1}$
}

Received: 6 September 2021 / Accepted: 6 September 2021 / Published online: 5 November 2021

(c) The Society of Polymer Science, Japan 2021

We are pleased to announce the winners of Rising Stars in Polymer Science 2021 as young influential. Polymer Journal has been enriched by the complex of wonderfully talented and diverse groups of these young scholars in addition to outstanding teams of well-established senior researchers. They bring a variety of new insights, both personal and professional, to the task of better understanding polymer science and engineering. Here they provide us with an array of novel observations drawn from such disciplines as synthesis, structure, and physical properties and functions and applications. We believe our readers will appreciate the opportunity to learn new voices in this special issue.

\section{Yoshifumi Amamoto \\ Kyushu University}

Yoshifumi Amamoto is an Assistant Professor in Institute for Materials Chemistry and Engineering, Kyushu University, Japan. He received his Ph.D. degree in Graduate School of Engineering from Kyushu University in 2011. He worked at Carnegie Mellon University in the USA as overseas research fellowships, JSPS in 2011-2012. After that, he was a postdoctoral fellow at the University of Tokyo, and Nagoya University, partially supported by research fellowships for young scientists (SPD), JSPS. He moved to his current position in 2018. He received SPSJ award for the outstanding paper in Polymer Journal sponsored by ZEON in 2010 and the award for encouragement of research in polymer science; SPSJ in 2020. His research interest is an integrated area between polymer science and data science. Especially, he has

Supplementary information The online version contains supplementary material available at https://doi.org/10.1038/s41428021-00559-y.

\footnotetext{
Keiji Tanaka

hpj@spsj.or.jp

1 Professor, Kyushu University, Fukuoka, Japan
}

engaged in structure-property relationships of elastomers and crystalline polymers in consideration of their higherorder structure.

\section{Masayuki Gon \\ Kyoto University}

Masayuki Gon earned his Ph.D. in Polymer Chemistry from Kyoto University in 2016. He developed threedimensionally arranged $\pi$-conjugated materials and studied their specific optical and physical properties based on structure and chirality. He worked as a visiting research fellow in the group of Prof. Kenneth J. Wynne at Virginia Commonwealth University in America, in 2014. Now, he has been an Assistant Professor of the Department of Polymer Chemistry, Graduate School of Engineering, Kyoto University since 2016. His present research theme is the development of functional $\pi$-conjugated materials focused on the unique nature of heteroatoms.

\section{Shinichi Hata \\ Sanyo-Onoda City University}

Shinichi Hata obtained his Ph.D. under the supervision of Prof. Yukishige Kondo from the Tokyo University of Science in 2014 in Colloid and Interface Science. He started working as a postdoctoral fellow in the Laboratory of Prof. Miho Yamauchi at the World Premier International (WPI) International Institute for Carbon-Neutral Energy Research ( ${ }^{2}$ CNER) at Kyushu University. Since 2017, he has worked as a Project Assistant Professor in the Laboratory of Prof. Masatake Haruta at Research Center for Gold Chemistry, Graduate School of Urban Environmental Sciences, Tokyo Metropolitan University. He became an Assistant Professor in the Department of Applied Chemistry, Faculty of Engineering, Sanyo-Onoda City University from April 2018. His current research interests are the development of organic and hybrid thermoelectric materials based on polymers and carbon materials, and the research conducted in collaboration with Prof. Yukihide Shiraishi and Prof. Naoki Toshima. His major interests are the fabrication and application of novel functional nanostructures based on nanomaterial and 
porous material. He was awarded The 50th Kurata Grants (2018).

\section{Yu-I Hsu \\ Osaka University}

Yu-I Hsu is an Assistant Professor in the Department of Applied Chemistry in the Graduate School of Engineering at Osaka University. She received her Ph.D. under the supervision of Prof. Yoshiharu Kimura from the Department of Biobased Materials Science at Kyoto Institute of Technology in 2015. She was a postdoctoral researcher at the Department of Biomedical Engineering, National Cerebral and Cardiovascular Center Research Institute from 2015 to 2019. She is particularly interested in polymer synthesis, polymer chemistry, bioplastic, biomaterials, such as injectable biodegradable hydrogels. Recently her research is focused on the novel surface oxidation method of engineering plastic using chlorine dioxide radical, to introduce functional groups for more application. Her research is also focused on the dual responsive hydrogel system with a rapid gelation rate and excellent toughness to achieve the applications in tissue engineering scaffolds.

\section{Ryohei Kakuchi \\ Gunma University}

Ryohei Kakuchi received his Ph.D. degree from the Hokkaido University in 2009 with financial support from a Japan Society for Promotion of Science (JSPS) research fellowship. After the Ph.D., he moved to Germany and started postdoctoral works at Martin-Luther University, University of Mainz, and the University of Hamburg from 2009 to 2014, partly supported by JSPS research fellowships. Then, he was appointed as a Research Assistant Professor at Kanazawa University in 2014. Based on the program entitled the Leading Initiative for Excellent Young Researchers (LEADER), he was then appointed as an Assistant Professor at Gunma University in 2017. His major interests are the development of novel polymer synthesis based on unique organic transformation reactions including multi-component reactions. He received the Award for Encouragement of Research in Polymer Science; The Society of Polymer Science, Japan (2017).

\section{In-Hwan Lee Ajou University}

In-Hwan Lee received his B.S. (2010) and Ph.D. (2016) degrees from the Seoul National University under the supervision of Prof. Tae-Lim Choi. Thereafter, he worked as a postdoctoral fellow with Prof. Craig J. Hawker in the Materials Research Laboratory at the University of California, Santa Barbara. Since 2018, he has been an Assistant Professor in the Department of Chemistry at Ajou University, South Korea. His research interests are the development of new synthetic strategies for functional soft materials and their application in energy harvesting, plastic recycling, and bio-health care.

\section{Hua Lu \\ Peking University}

Hua Lu is currently an Associate Professor in the College of Chemistry and Molecular Engineering, Peking University. He earned his B.Sc. in Chemistry from Peking University in 2006 and Ph.D. in Materials Science and Engineering from the University of Illinois at Urbana-Champaign under the supervision of Prof. Jianjun Cheng in 2011. He worked as a Damon Runyon Cancer Research Foundation postdoctoral fellow at the Scripps Research Institute (La Jolla, CA) with Prof. Peter G. Schultz. He started his independent research in 2014 as a Tenure-track Assistant Professor at Peking University, where he was promoted to Associate Professor with tenure in January 2020. His research focuses on the development of synthetic methodologies for novel polymeric biomaterials such as polypeptides and polyesters, synthesis of recyclable polymers for sustainability, and protein conjugation for therapeutics/catalysis/self-assembly. $\mathrm{He}$ is a recipient of ACS AkzoNobel Award for Outstanding Graduate Research in Polymer Chemistry (2013), Honorable Mention of IUPAC Prize for Young Chemists (2012), Excellent Young Investigator of NSFC (2017), Chinese Chemical Society (CCS) Prize for Young Scientists (2017), and CCS Polymer Division Young Scholar Award (2019). He has served as an Associate Editor for Biomacromolecules (ACS) since April 2020.

\section{Tsuyoshi Minami \\ The University of Tokyo}

Tsuyoshi Minami obtained his Ph.D. degree from Tokyo Metropolitan University in 2011. During his Ph.D. research, he worked at the University of Bath as a collaborative researcher. Between 2011 and 2013, he was a Postdoctoral Research Associate at Bowling Green State University. In 2013, he was appointed as a Research Assistant Professor at the same university. Thereafter, he proceeded to Yamagata University as an Assistant Professor in 2014. He was appointed as a Lecturer at the University of Tokyo in 2016, and then he has been an Associate Professor since 2019 at the same university. His research interests are supramolecular analytical chemistry and self-assembled materials.

\section{Tsuyoshi Nishikawa Kyoto University}

Tsuyoshi Nishikawa was born in 1989 in Nara, Japan. $\mathrm{He}$ received his bachelor and Ph.D. degrees from Kyoto University in 2012 and 2017, respectively, under the supervision of Prof. Michinori Suginome. In April of 2017, he began his academic career as a postdoctoral 
fellow in Prof. Shigehiro Yamaguchi's group at Nagoya University. In April of 2018, he joined Prof. Makoto Ouchi's laboratory at Kyoto University as an assistant professor. His research interest is novel polymer synthesis through monomer design based on the unique properties of the main-group element.

\section{Tomoki Nishimura \\ Shinshu University}

Tomoki Nishimura received his B. Eng., M. Eng., and Ph.D. Degrees under the supervision of Prof. Kazuo Sakurai from The University of Kitakyushu in 2010 in physical chemistry and supramolecular chemistry. He was a postdoctoral researcher (2010-2012) in the group of Prof. Tony D. James at the University of Bath (UK) and a program-specific researcher (2012-2018) in JST Akiyoshi bionanotransporter project. From 2018 to 2020, he was a program-specific assistant professor in the group of Prof. Kazunari Akiyoshi at Kyoto University. In 2020, he moved to Shinshu University as an assistant professor. His current research interests include the self-assembly of amphiphilic copolymers and the development of functional polymer materials for biomedical applications.

\section{Haritz Sardon \\ University of Basque Country}

Haritz Sardon has been an Assistant Professor at the University of Basque Country since 2018. He graduated from the University of Basque Country in 2011 with honors before joining the group of Dr. Hedrick at IBM-Almaden Research Center as a postdoc in 2012, where he spent 2 years. In 2014 he returned to Spain with a Spanish Ministry grant and joined POLYMAT as group leader. Haritz Sardon has participated in 105 peer-reviewed publications, with more than 50 as the corresponding author. The impact of his work can be measured by increasing the number of citations near 1000 in 2020 citations. He has been awarded several awards including the Excellence of Young Researcher in Chemistry Award by the Spanish Royal Society (2021) and the Excellence of Young Researcher in Polymers Award by the Grupo Español de Polímeros (2020). His overall research aims to prepare new functional polymeric materials using sustainable polymerization processes. Specifically, his investigations involve the use of green polymerization processes such as monomers from plastic recycling, reagents from renewable sources or the use of less hazardous organocatalysts.

\section{Akira Takahashi \\ Kanagawa University}

Akira Takahashi received his B.S. from Tokyo University of Science under the supervision of Prof. Takanori Matsuda in 2010, and M.S. from Tokyo Institute of
Technology (Tokyo Tech) under the supervision of Prof. Takanori Fukushima in 2013. In the same year, he joined Hitachi Chemical Co., Ltd. He then entered the doctoral course of the Department of Organic and Polymeric Materials at Tokyo Tech in October 2014. He received his Ph.D. from Tokyo Tech under the supervision of Prof. Hideyuki Otsuka in 2017. He was a Japan Society for the Promotion of Science (JSPS) research fellow (DC2) from April to September 2017, and subsequently started his academic career at Kanagawa University as an assistant professor in the group of Prof. Atsushi Kameyama in 2017. His research interests cover hetero compounds, functional polymers, and supramolecules.

\section{Kenji Takada \\ Japan Advanced Institute of Science and Technology}

Kenji Takada is an Assistant Professor of Japan Advanced Institute of Science and Technology (JAIST), Ishikawa in Japan. He received his Ph.D. in Hokkaido University in precise polymer synthesis using eco-friendly organocatalysts. He was subsequently appointed as a Japan Society for the Promotion of Science fellow as DC1 and PD at Hokkaido University. From 2015 to 2016, he was appointed as a Research Assistant Professor of Center of Innovation Project (Japan Science and Technology Agency, JST) in Kanazawa University. From 2016 to 2020, he joined to JAIST as a research assistant professor. In 2020, he moved to Green Science Material Inc. as a researcher. Since 2021, he has been in his current position at the JAIST. His research interests are in the development of highperformance and functional materials using organic synthesis and controlled polymerization. In particular, he has been actively studying the cinnamate-based polymers for high-thermal resistant and photo-functional application.

\section{Ryo Tanaka \\ Hiroshima University}

Ryo Tanaka was born in Wakayama Prefecture, Japan. He received his Ph.D. degree from the University of Tokyo under the guidance of Prof. Kyoko Nozaki in 2011. After working as a postdoctoral fellow of Humboldt-universtät zu Berlin at the group of Prof. Stefan Hecht, he joined Hiroshima University as an assistant professor in June 2012. He has promoted to associate professor, his current occupation, in April 2021. He received Award for Encouragements of Research in Polymer Science from The Society of Polymer Science, Japan in 2019. His current research interests are the development of the transition-metal based catalyst system for precise coordination polymerization.

Publisher's note Springer Nature remains neutral with regard to jurisdictional claims in published maps and institutional affiliations. 